\title{
Synthetic NPA diagnostic for energetic particles in JET plasmas
}

\author{
J. Varje, ${ }^{a, 1}$ P. Sirén, ${ }^{b}$ H. Weisen, ${ }^{c}$ T. Kurki-Suonio, ${ }^{a}$ S. Äkäslompolo ${ }^{a, 2}$ and JET contributors ${ }^{3}$ \\ ${ }^{a}$ Department of Applied Physics, Aalto University, P.O. Box 11100, 00076 AALTO, Finland \\ ${ }^{b}$ VTT Technical Research Centre of Finland, P.O. Box 1000, 02044 VTT, Finland \\ ${ }^{c}$ SPC, station 13, EPFL, 1015 Lausanne, Switzerland \\ E-mail: jari.varje@aalto.fi
}

AbstRact: Neutral particle analysis (NPA) is one of the few methods for diagnosing fast ions inside a plasma by measuring neutral atom fluxes emitted due to charge exchange reactions. The JET tokamak features an NPA diagnostic which measures neutral atom fluxes and energy spectra simultaneously for hydrogen, deuterium and tritium species. A synthetic NPA diagnostic has been developed and used to interpret these measurements to diagnose energetic particles in JET plasmas with neutral beam injection heating.

The synthetic NPA diagnostic performs a Monte Carlo calculation of the neutral atom fluxes in a realistic geometry. The 4D fast ion distributions, representing NBI ions, were simulated using the Monte Carlo orbit-following code ASCOT. Neutral atom density profiles were calculated using the FRANTIC neutral code in the JINTRAC modelling suite. Additionally, for rapid analysis, a scan of neutral profiles was precalculated with FRANTIC for a range of typical plasma parameters. These were taken from the JETPEAK database, which includes a comprehensive set of data from the flat-top phases of nearly all discharges in recent JET campaigns.

The synthetic diagnostic was applied to various JET plasmas in the recent hydrogen campaign where different hydrogen/deuterium mixtures and NBI configurations were used. The simulated neutral fluxes from the fast ion distributions were found to agree with the measured fluxes, reproducing the slowing-down profiles for different beam isotopes and energies and quantitatively estimating the fraction of hydrogen and deuterium fast ions.

KeYwords: Nuclear instruments and methods for hot plasma diagnostics, simulation methods and programs, analysis and statistical methods

\footnotetext{
${ }^{1}$ Corresponding author.

${ }^{2}$ Current address: Max-Planck-Institut für Plasmaphysik, Wendelsteinstr. 1, 17491 Greifswald, Germany

${ }^{3}$ See the author list of "Overview of the JET results in support to ITER" by X. Litaudon et al. to be published in Nuclear Fusion Special issue: overview and summary reports from the 26th Fusion Energy Conference (Kyoto, Japan, 17-22 October 2016)
} 


\section{Contents}

1 Introduction 1

2 Synthetic NPA model $\quad 1$

3 Results 3

4 Conclusion 5

\section{Introduction}

Neutral particle analysis (NPA) is a diagnostic method for measuring neutral atom emissions resulting from charge exchange $(\mathrm{CX})$ reactions between ions and neutral particles in a fusion plasma. The neutralized ions can escape the confining magnetic field and carry with them information on the ion population. However, the measured signal only represents the line-integrated energy spectrum of the emitted atoms. Thus synthetic diagnostics are required for inferring properties of the original ion distribution from the measurements. Of particular interest is analysis of fast ions, such as those resulting from neutral beam injection (NBI) heating, as only few diagnostics [1-4] can directly measure properties of these particles.

The JET tokamak is equipped with a low-energy neutral particle analyzer [5] which measures fluxes and energy spectra simultaneously for neutral hydrogen, deuterium and tritium along a radial sightline on the midplane of the tokamak (figure 1). Incoming neutral particles are ionized in a carbon stripping foil, after which they are accelerated and separated using parallel magnetic and electric fields. The particles are then measured with three rows of scintillator detectors, each corresponding to one isotope and energy. The diagnostic can measure multiple energy ranges from $4 \mathrm{keV}$ upwards. For measurements suitable for NBI ions at JET, the energy ranges are 4-97 keV for hydrogen, 5-41 keV for deuterium and 5-22 keV for tritium.

In this contribution we describe a synthetic NPA model for the JET low-energy neutral particle analyzer [6], and present results from modelling the fast ion contribution to the NPA signal in neutral beam injection heated discharges in the 2016 JET hydrogen campaign [7], where simultaneous hydrogen and deuterium injection was applied in plasmas with various hydrogen concentrations.

\section{Synthetic NPA model}

The synthetic NPA diagnostic calculates the line-averaged neutral atom flux

$$
\Gamma=\int_{0}^{L} n_{i} n_{0}\langle\sigma v\rangle_{\mathrm{CX}} e^{-\int_{0}^{l} \frac{n_{e}\langle\sigma v\rangle_{\text {ion }}}{v}} d l^{\prime} d l
$$

where $n_{e}, n_{i}$ and $n_{0}$ are the electron, ion and neutral densities, $v$ is the velocity of the fast neutral and $\langle\sigma v\rangle_{\mathrm{CX}}$ and $\langle\sigma v\rangle_{\mathrm{ion}}$ are the charge exchange and ionization rate coefficients between the fast particle 

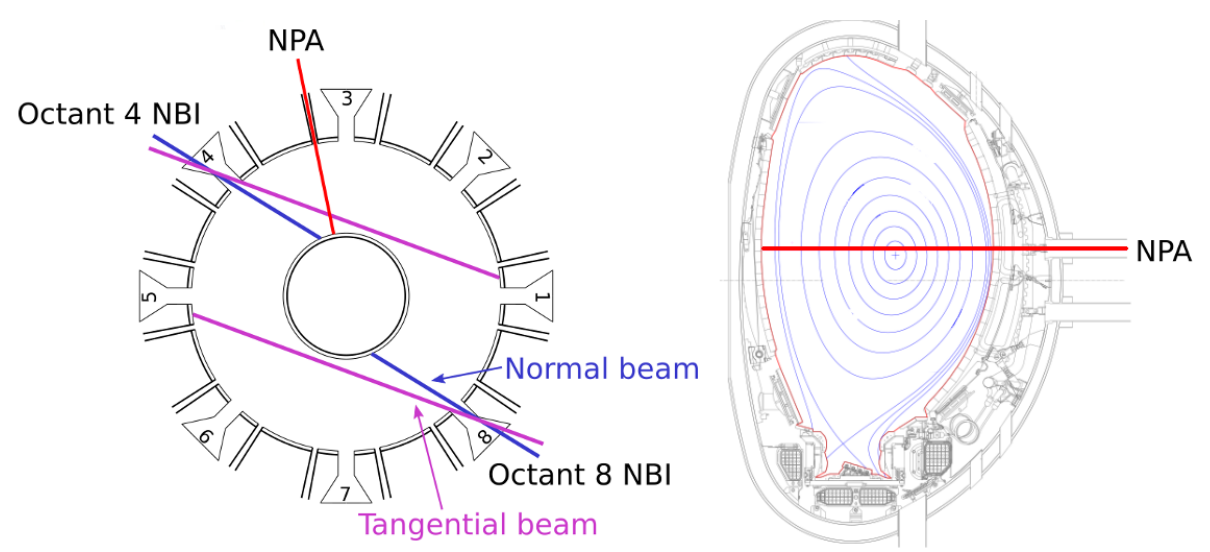

Figure 1. JET low-energy NPA line of sight in a horizontal (left) and poloidal cross section (right).

and a Maxwellian background. The outer integral evaluates the neutral source along the viewing chord through the plasma with length $L$, while the exponential term accounts for the attenuation of the neutral flux due to re-ionization along the chord from the source to the edge of the plasma.

The flux is integrated using a Monte Carlo method where particles are sampled in the diagnostic viewing cone, and the contribution visible to the detector is calculated. The local charge exchange rate is evaluated based on a 4D fast ion distribution and 1D neutral density, after which the attenuation of the neutral flux is calculated as a line integral from the point of origin to the detector. ADAS [8] $\mathrm{CX}$ and beam-stopping coefficients were used for the charge exchange and ionization rates.

The necessary input data for the synthetic diagnostic were produced using a suite of plasma physics codes and databases (figure 2). Plasma density and temperature profiles were obtained from the JETPEAK database, previously used for transport and neutron deficit studies at JET [9]. The database contains a comprehensive set of data, including basic plasma parameters and profiles, averaged over stationary phases of discharges in recent JET campaigns. The profiles, together with EFIT [10] reconstructed equilibria, were used in the Monte Carlo orbit-following code ASCOT [11] to simulate the 4D slowing-down distribution of the NBI ions. An ensemble of 100000 test particles was used in the simulations, generated with the beamlet-based Monte Carlo NBI ionization code BBNBI [12].

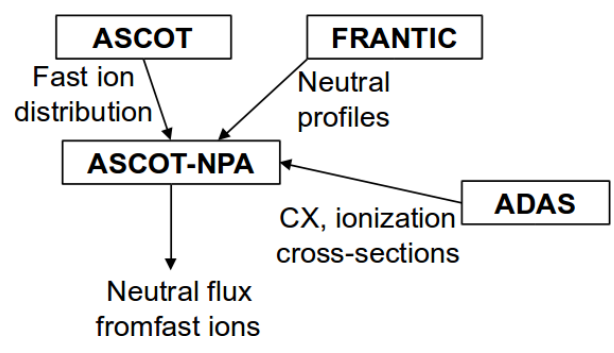

Figure 2. The suite of codes used to simulate the NPA signal.

Neutral density profiles were pre-calculated with the 1D neutral code FRANTIC [13] in the JINTRAC code suite for a range of typical plasma parameters, covering most of the discharges in the hydrogen campaign (figure 3). Electron and ion density and temperature profiles from the JETPEAK 
database were averaged from discharges with core densities of 2, 3, 4 and $5 \cdot 10^{19} \pm 10 \% \mathrm{~m}^{-3}$ and temperatures of $1,2,3$ and $4 \pm 10 \% \mathrm{keV}$ to arrive at representative profiles to be used in the FRANTIC simulations. Approximately 10-50 discharges were included in the averages for each density and temperature. The pre-calculated neutral density profiles were then interpolated for each of the simulated cases.

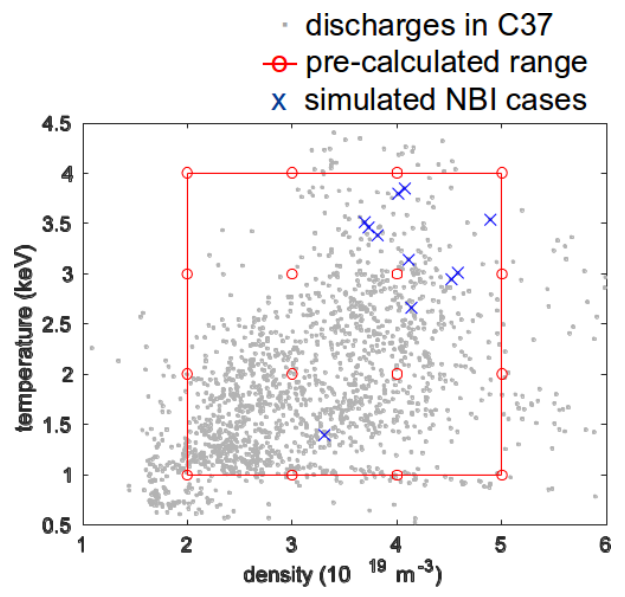

Figure 3. Operating space of the precalculated neutral profiles together with the hydrogen campaign discharges (grey), with the mixed NBI discharged simulated in this work highlighted (blue).

\section{Results}

The synthetic NPA diagnostic was applied to a set of JET discharges featuring simultaneous hydrogen and deuterium NBI heating during the hydrogen campaign. Some of the discharges were simulated at multiple time points with different NBI heating power. The total NBI power in the simulated cases varied between 1.9-13.1 MW, while the power fraction between the beam isotopes $P_{\mathrm{H}-\mathrm{NBI}} / P_{\mathrm{D}-\mathrm{NBI}}$ varied between $22-83 \%$. The bulk hydrogen concentration $n_{\mathrm{H}} /\left(n_{\mathrm{H}}+n_{\mathrm{D}}\right)$ varied between $12-83 \%$.

The simulated fluxes were fitted to the measurements by scaling the neutral density profiles by up to $65 \%$ with the assumption that the largest uncertainty is due to the simulated recycling neutral profiles. The fitting was done at $E>20 \mathrm{keV}$, corresponding to the fast ion energies, simultaneously for both isotopes, thus maintaining the relative fluxes and profiles.

The simulated neutral fluxes were found to generally agree with the measurements in the range $E>20 \mathrm{keV}$ (figure 4). In all cases, the model reproduces both the different slowing-down profiles of the $80 \mathrm{keV} \mathrm{H}$ beams and the $120 \mathrm{keV} \mathrm{D}$ beams, including the step at the $40 \mathrm{keV}$ half-energy injection for the $\mathrm{H}$ beam. Additionally, the simulated fluxes correctly model the fraction of fast $\mathrm{H}$ and $\mathrm{D}$ atoms, corresponding to the different injection powers of the two isotopes.

The largest differences were be observed at low NBI power of 0-2 MW and high bulk hydrogen concentrations above $75 \%$. This is likely due to the neutral fluxes from the thermal bulk ions, which begin to dominate at energies $E<20 \mathrm{keV}$. Additionally, the fitting procedure used only assumes scaling of the neutral density, whereas also the penetration of the neutrals can vary depending on the exact plasma conditions. 

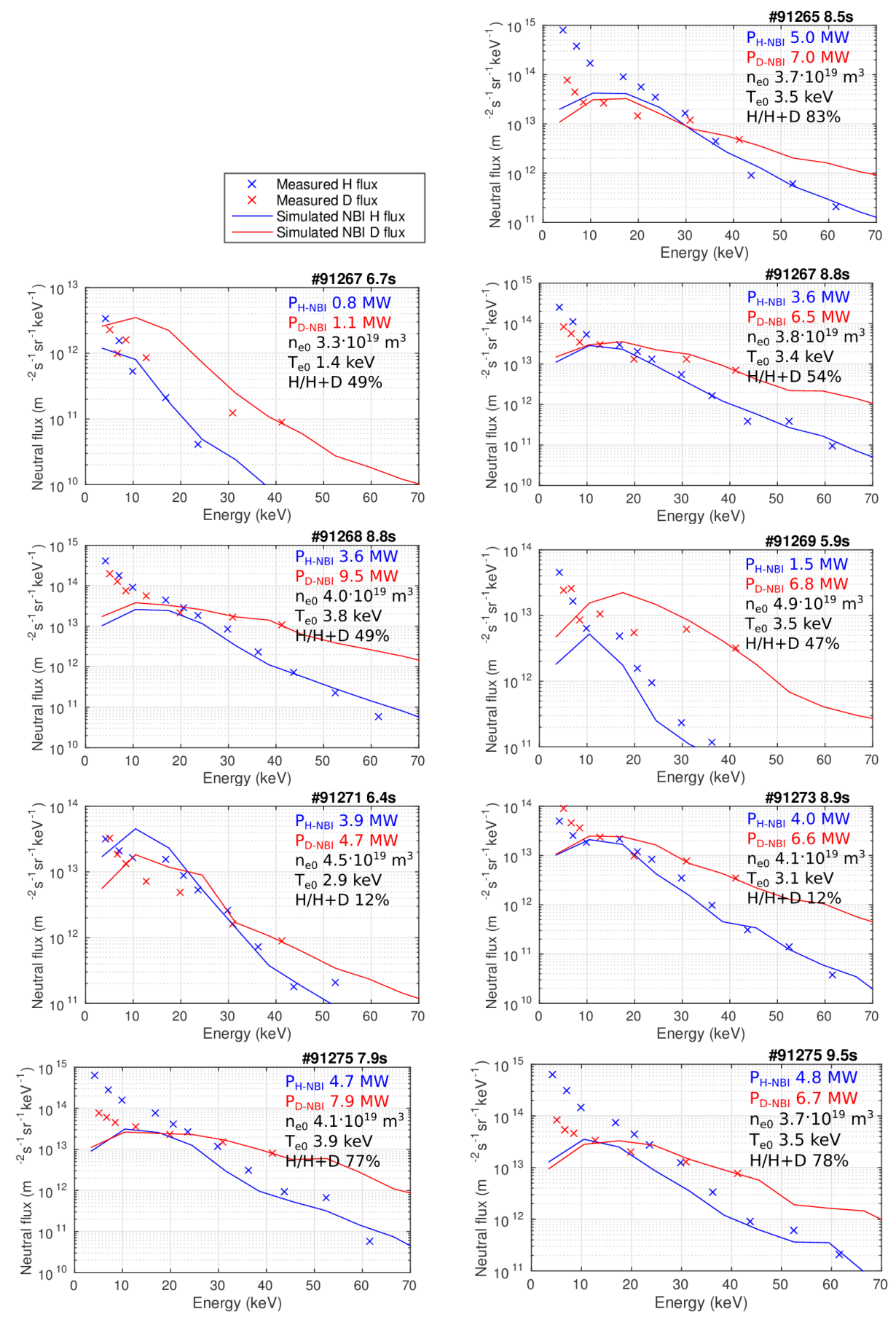

Figure 4. Measured and synthetic NPA fluxes for discharges with simultaneous hydrogen and deuterium NBI injection, together with the $\mathrm{H}$ and D NBI powers $P_{\mathrm{H}-\mathrm{NBI}}$ and $P_{\mathrm{D}-\mathrm{NBI}}$, core density $n_{e 0}$ and temperature $T_{e 0}$ and bulk hydrogen fraction $n_{H} /\left(n_{H}+n_{D}\right)$. 


\section{Conclusion}

The synthetic NPA diagnostic for NBI ion slowing-down measurements agrees with the measured fluxes for the fast ion contribution in hydrogen-deuterium discharges, with $\mathrm{H}$ and D NBI power between 2-13 MW and hydrogen concentrations ranging from $12 \%$ to $83 \%$. The simulated fluxes reproduce the experimentally observed slowing-down profiles and beam ion isotope fractions. However, some uncertainties remain at the extreme ends of the simulated operating space.

The results from the mixed NBI discharges, simultaneously reproducing the slowing-down of different isotopes, demonstrates the validity of the presented rapid analysis procedure for synthetic NPA. The procedure is planned to be applied to all discharges with sufficient measurements in the hydrogen campaign, including discharges with only $\mathrm{H}$ and $\mathrm{D}$ injection, enabling a systematic study of the NBI distribution functions in various JET plasmas.

The model will be expanded to also cover the JET high-energy NPA, which is typically used in to measure MeV-range particles due to ion cyclotron radiofrequency heating (ICRH). This will be important for validation of ICRH heating codes such as RFOF-ASCOT [14]. The model will also be used in support of thermal ion isotope ratio measurements using the NPA, where the NBI fluxes need to be accounted for when inferring bulk isotope ratios from the measurements [6], together with extending the model to simulate the neutral fluxes from the Maxwellian distribution of the thermal ions.

\section{Acknowledgments}

This work has been carried out within the framework of the EUROfusion Consortium and has received funding from the Euratom research and training programme 2014-2018 under grant agreement No 633053. The views and opinions expressed herein do not necessarily reflect those of the European Commission. The work was partially funded by the Academy of Finland project No.298126. Some of the calculations were performed using computer resources within the Aalto University School of Science 'Science-IT' project.

\section{References}

[1] Bindslev H. et al., Physical Review Letters, 83 (1999) 3206

[2] Geiger B. et al., Plasma Physics and Controlled Fusion, 53 (2011) 065010

[3] Hellesen C. et al., Plasma Physics and Controlled Fusion, 52 (2010) 085013

[4] Nocente M. et al., Nuclear Fusion, 54 (2014) 104010

[5] Afanasyev V.I. et al., Review of Scientific Instruments 74 (2003) 2338

[6] Varje J. et al. 43rd EPS conference on Plasma Physics, O5.131, 4-8 July 2016, Leuven, Belgium

[7] Nunes I. et al. 26th IAEA Fusion Energy Conference, PDP-2, 17-22 October 2016, Kyoto, Japan

[8] ADAS Atomic Data and Analysis Structure, http://www.adas.ac.uk

[9] Weisen H. et al., Nuclear Fusion, 57 (20179 076029

[10] Lao L. et al., Nuclear Fusion, 25 (1985) 1611 
[11] Hirvijoki E. et al., Computer Physics Communications, 185 (2014) 1310-1321

[12] Asunta O. et al., Computer Physics Communications, 188 (2015) 33-46

[13] Tamor S., SAI Report 023-79-1056LJ

[14] Johnson T. et al., AIP Conference Proceedings, 1406 (2011) 373 